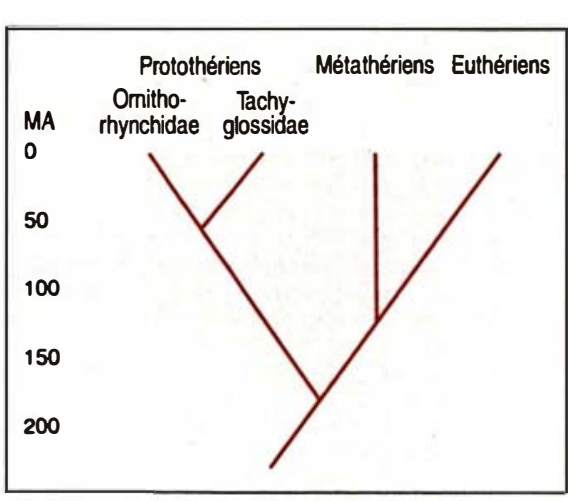

Figure 1. Relations entre les groupes de mammifères. Euthériens (mammifères "à placenta "); Métathériens (marsupiaux); Protothériens (monotrèmes), divisés en deux familles; MA, millions d'années. (D'après [1].)

priées, et en validant les résultats en vérifiant que les sondes tirées du bras long de l'X se lient au chromosome $\mathrm{X}$ de l'ornithorynque.

Les sondes suivantes ont été utilisées : DMD (dystrophine), OTC (ornithine transcarbamylase), SYN1 (synapsine 1), ZFX, MAOA (monoamine oxydase A), POLA (DNA polymérase A), CYBB (cytochrome b, polypeptide $\beta$ ). Elles sont toutes autosomiques comme chez les marsupiaux. L'hypothèse proposant que la région qui porte ces gènes ait été perdue par l'X chez les marsupiaux devient difficilement tenable, puisque la divergence des monotrèmes a précédé celle des marsupiaux, et cela impliquerait que la même région ait été perdue deux fois de façon indépendante. Il est donc plus probable que l'ancêtre commun des marnmifères ne portait pas cette région sur l'X, qui l'a acquise chez les euthériens.

De quels chromosomes proviennent donc ces gènes? Il en existe deux groupes, l'un sur le bras long du chromosome 1, l'autre sur le bras court du chromosome 2 ; de même, chez les marsupiaux, on les trouve groupés sur les bras courts des chromosmes 1 et 5 . Il y a donc eu probablement fusion de deux blocs, qui se sont ensuite liés au chromosome $\mathrm{X}$. Une autre question, non encore résolue, est de comprendre comment des gènes autosomiques, donc présents en deux doses, ont acquis le processus d'inactivation nécessaire à la compensation entre les deux sexes. L'hypothèse proposée est que ces gènes étaient d'abord dans une zone pseudo-autosomique à la fois sur l'X et l'Y, puis que se sont produites une amputation de l'Y et une inactivation concomitante sur l'X.

De telles observations pourraient favoriser des recherches concernant des protéines dont on suppose qu'elles dérivent d'un ancêtre commun. On pourrait par exemple se demander si, il y a $200 \mathrm{MA}$, les gènes de la dystrophine et de la DRP (dystrophin related protein) siégeaient au même endroit ou même étaient confondus.

Remarquons enfin que, si nous quittons les mammifères pour nous référer par exemple aux oiseaux, les gènes portés par les chromosomes $\mathrm{X}$ des mammifères ne se trouvent pas sur les chromosomes sexuels $\left(\mathrm{m} / \mathrm{s} n^{\circ} 1, \mathrm{vol} .7\right.$, p. 76).

\section{J.-C. D.}

1. Watson JM, Spencer JA, Riggs AD, Marshall Graves JA. Sex chromosome evolution Platypus gene mapping suggests that part of the human X chromosome was originally autosomal Proc Natl Acad Sci USA 1991; 88 : 11256-60.

\title{
BRĖVES
}

Phosphorylation de facteurs nucléaires par des récepteurs membranaires à activité tyrosine kinase. Les détails de la transduction d'un signal mitogénique depuis la membrane cellulaire, qui perçoit un facteur de croissance, jusqu'au noyau, dans lequel l'activité transcriptionnelle de nombreux gènes est modifiée, restent incomplètement connus. L'un des mécanismes qui a été récemment très étudié est celui de la translocation induite, sous l'effet d'une phosphorylation dépendant de la protéine kinase C ou de la protéine kinase A (stimulée par l'AMP cyclique), d'un facteur transcriptionnel du cytoplasme vers le noyau $\left(\mathrm{m} / \mathrm{s} n^{\circ} 8\right.$, vol. 6, p. $803 ; n^{\circ} 1$, vol. $7, p .67)$. Une équipe de chercheurs américains de New Haven, CT et de Charleston, SC, vient maintenant de montrer que la reconnaissance par son récepteur du facteur IGF-1 (insulin growth factor 1) s'accompagnait d'une augmentation de la phosphorylation sur des tyrosines de protéines nucléaires, notamment de c-Jun [1]. Le récepteur IGF-1 appartient à la même famille que celui de l'insuline et de nombreux autres facteurs de croissance : il s'agit d'une molécule membranaire dont l'extrémité carboxy-terminale intracytoplasmique possède un domaine tyrosine kinase stimulé par la liaison du ligand. Les mécanismes de la phosphorylation sur des tyrosines des protéines nucléaires restent inconnus puisqu'il n'est pas très probable que, après endocytose, le récepteur/tyrosine kinase aille dans le noyau, quoiqu'un tel résultat ait été proposé par certains auteurs, mais démentis par d'autres. L'activation de l'activité kinasique du récepteur pourrait, en réalité, déclencher une cascade d'activations de tyrosine kinases. La protéine kinase c-Abl est, ainsi, à localisation nucléaire et pourrait, par exemple, servir d'intermédiaire entre la membrane et le noyau. Ces résultats sont très préliminaires puisqu'on ne sait rien des conséquences fonctionnelles de la phosphorylation sur une tyrosine de facteurs de transcription, notamment de c-Jun, et que les mécanismes de cette phosphorylation restent spéculatifs. Il s'agit cependant là d'une nouvelle piste à explorer pour expliquer la transmission vers le noyau de l'information reçue par un récepteur membranaire avec activité de tyrosine kinase.

[1. Oemar BS, et al. J Biol Chem $1991 ; 266$ : 24241-4.] 